\title{
„Durch die List ist der Willen zum Weiblichen geworden." Bemerkungen zu einer Stelle aus Hegels Jenaer Systementwürfen
}

"Par la ruse, la volonté est devenue le féminin. " Remarques à propos d'un passage dans les Esquisses de système d'Iéna de Hegel

"Through cunning, the will becomes feminine." Comments on a passage in Hegel's Jena System Sketches

\section{Friederike Kuster}

\section{OpenEdition}

Journals

Édition électronique

URL : http://journals.openedition.org/ceg/1520

DOI : $10.4000 /$ ceg. 1520

ISSN : 2605-8359

Éditeur

Presses Universitaires de Provence

Édition imprimée

Date de publication : 15 juin 2015

Pagination : $165-176$

ISBN : 978-2-85399-993-9

ISSN : 0751-4239

\section{Référence électronique}

Friederike Kuster, « „Durch die List ist der Willen zum Weiblichen geworden." Bemerkungen zu einer Stelle aus Hegels Jenaer Systementwürfen », Cahiers d'Études Germaniques [Online], 68 | 2015, Online erschienen am: 17 Dezember 2017, abgerufen am 01 Dezember 2020. URL : http://

journals.openedition.org/ceg/1520; DOI : https://doi.org/10.4000/ceg.1520 


\section{„Durch die List ist der Willen zum Weiblichen geworden. “ Bemerkungen zu einer Stelle aus Hegels Jenaer Systementwürfen}

Friederike KUSTER

Bergische Universität Wuppertal

Hegel hat in seinem Werk dem Geschlechtsunterschied und dem Verhältnis der Geschlechter immer wieder ausführliche Reflexionen gewidmet. Diese reichen von der Naturphilosophie in die Philosophie des Geistes. Angelegt und begründet in der organischen Natur erstreckt sich die geschlechtliche Polarität von männlich und weiblich bzw. von Mann und Frau bis in die Zweiteilung der sittlichen Welt in eine weibliche und eine männliche Sphäre, in Familie und Staat.

Neben den einschlägigen Passagen zum Geschlechtsverhältnis in der Naturphilosophie, wo es als Teil des Gattungsprozesses die höchste Darstellungsform der Vernunft in der Natur bildet, finden sich die ausführlichsten und systematisch ausgearbeiteten Darstellungen der Geschlechterrelation und der Familie in der Phänomenologie des Geistes von 1807 und in den Grundlinien der Philosophie des Rechts von 1820 .

Demgegenüber soll es allerdings hier um einen Text aus den Jenaer Systementwürfen III ${ }^{1}$ von 1805/06 gehen. Die verschiedenen Systementwürfe der Jenaer Jahre 1801 bis 1806 können gewissermaßen als - freilich der systematischen Anlage nach mehr oder weniger differierende - Vorstufen zur Phänomenologie des Geistes von 1807 betrachtet werden. Die Jenaer Systementwürfe III umfassen die Naturphilosophie und die Philosophie des Geistes. Der Satz, der im Titel genannt ist und um dessen Interpretation es im Weiteren geht, findet sich in der Philosophie des Geistes im Kontext eines von Hegel skizzierten Bildungsgangs des Bewusstseins in den Medien von Sprache, Arbeit und Familie.

„Durch die List ist der Willen zum Weiblichen geworden.“2 Diese Behauptung lässt stutzen, weckt die Neugier und den hermeneutischen Ehrgeiz. Hier ist nicht einfachhin von weiblicher List die Rede, sondern vielmehr, dass der Wille durch die List weiblich wird. Nicht also die Frau setzt die List in die Welt, sondern die List

1 Georg Wilhelm Friedrich Hegel, Jenaer Systementwürfe III/ Naturphilosophie und Philosophie des Geistes, neu hrsg. von Rolf-Peter Horstmann, Hamburg, Felix Meiner Verlag, 1987 (im Folgenden zitiert als J III).

2 Ibid., S. 190. 
- fragen ließe sich freilich welche List - erzeugt das Weibliche und noch dazu als das Weibliche am Willen. Das ist so nicht ohne weiteres nachzuvollziehen. Hinzu kommt noch, dass das Weibliche und die List einige Zeilen später in einen Bezug zum Begriff des Bösen gesetzt werden. Der ganze Gedankengang schließlich wird in starker Verknappung und auf wenigen Seiten, zudem in einem fragmentarischen Duktus entwickelt und präsentiert sich dementsprechend spröde.

Es ist erstaunlich, dass dieser Konnex von Weiblichem, List und Bösem, der unvermeidlich die Assoziation religiöser abendländischer Topoi evoziert, im Vorlesungsfragment von 1805/06 in einen Kontext gestellt ist, der demgegenüber zunächst eher abseitig erscheint: es geht, lapidar gesagt, um die Erfahrung, die das praktische Bewusstsein im Prozess der Arbeit macht. Überdies ist es bemerkenswert, dass dieser Hegel-Stelle, genauer dem Zusammenhang von List, Weiblichem und Bösem bislang noch keine Aufmerksamkeit geschenkt wurde, wobei freilich die Literatur zu Hegel, auch die zum frühen Hegel, Legion ist. Indes überrascht diese offenkundige Lücke auch nicht allzu sehr, denn die Themen des Geschlechts und des Geschlechterverhältnisses werden in der philosophischen Rezeption chronisch stiefväterlich behandelt, zumeist in ihrer systematischen Relevanz sogar komplett ignoriert oder übergangen.

Vor diesem Hintergrund bietet es sich an, diesem Textabschnitt erstmalig eine genaue Lektüre zu widmen. Dabei ist zunächst der Kontext der Überlegungen zur List und zum Weiblichen zu explizieren und eine Rekonstruktion des Gedankenganges zu leisten, der List und Weibliches gleichsam als Resultate der menschlichen Arbeit gewinnt. Im zweiten Schritt ist zu klären, inwiefern der weibliche Charakter des Willens auch als das Böse verstanden werden kann, wobei zu einer umfassenderen Interpretation des Begriffs des Bösen auf Hegels Vorlesungen zur Philosophie der Religion zurück gegriffen wird. Schließlich ist noch ein Blick zu werfen auf die veränderte Fassung des Gedankens der „weiblichen List“ und auf die systematisch andere Verortung in der Phänomenologie des Geistes. Die List nimmt dort im Rahmen einer Konzeption antiker Sittlichkeit die Gestalt der Intrige der Frauen an und wird zur schicksalshaften „Ironie“ des griechischen Staates.

\section{Arbeit - List - Weibliches}

Habermas hat in einer berühmten Interpretation der Jenaer Systemfragmente ${ }^{3}$ herausgestellt, wie Hegel für den Bildungsprozess des Geistes eine besondere, später aufgegebene Systematik zugrunde legt, in welcher:

Die Kategorien Sprache Werkzeug und Familie [...] drei gleichwertige Muster dialektischer Beziehung [bezeichnen]: die symbolische Darstellung, der Arbeitsprozess und die Interaktion auf der Grundlage der Reziprozität vermitteln Subjekt und Objekt je auf ihre Weise. ${ }^{4}$

3 Jürgen Habermas, Arbeit und Interaktion, in ders., Technik und Wissenschaft als Ideologie, Frankfurt a. M., Suhrkamp Verlag, 1969, S. 9-47.

4 Ibid., S. 9f. 
Die Bildung des Individuums erscheint hier an bestimmte Medien gebunden: an Gedächtnis, Arbeit und Familie nach der subjektiven Seite hin, die sich in ihren verobjektivierten Formen als Sprache, Werkzeug und Familiengut darstellen. Dass der Entwicklungsprozess des Geistes an Medien, an „Mitten“ gebunden ist, besagt, dass das Individuum sich vermittels, gleichsam inmitten der Potenzen von Sprache, Arbeit und Interaktion herausbildet. ${ }^{5}$ Dieser Zusammenhang kann hier nur im Umriss angedeutet werden. Zur Einordnung des hier interessierenden Textabschnitts ist entscheidend, dass er den systematischen Übergang von der Arbeit zur Familie stiftet. Verkürzt gesagt handelt es sich um einen Passus über den Charakter, den Hegel als den Willen bestimmt, sofern er seinen eigenen Gegensatz in sich enthält. Die kontrastierende Konstellation, welche die entzweiten Momente des Geistes im Charakter bilden, bringt Hegel dann in Zusammenhang mit dem Geschlechtsunterschied, der im Weiteren die Basis für die Familie abgibt. ${ }^{6}$

Diese innere Spaltung des Willens ist nun genauer zu explizieren. Einzusetzen ist dort, wo der Geist sich äußerlich verwirklicht und manifestiert, als Wille, als praktischer Geist. Die grundlegende Tätigkeit des praktischen Geistes ist Stillung der menschlichen Begierde. Das individuelle vereinzelte Bewusstsein ist dabei bezogen auf ein ,,absolut Entgegengesetztes, ein totes Ding ${ }^{67}$. Allerdings befriedigt der Mensch seine Begierde in grundsätzlich anderer Art als das Tier, insofern er arbeitet. In der Arbeit wird der menschliche Trieb zur „gehemmten Begierde“, zu einer Begierde, die ihre Stillung zunächst aufschiebt und den Gegenstand nicht vernichtet, sondern ihn gestaltet und sich damit in ihm realisiert.

Diese bildende Potenz der Arbeit wird in der Phänomenologie des Geistes im Rahmen des berühmten Kapitels zu Herrschaft und Knechtschaft herausgestellt: „Arbeit ist gehemmte Begierde, aufgehaltenes Verschwinden, oder sie bildet. “8 Arbeit bedeutet eine Formung nach zwei Seiten: sowohl nach der Seite des Gegenstands wie nach der des Arbeitenden, beide gewinnen vermittels der Arbeit Gestalt. Der Gegenstand verliert seine ursprüngliche Kontur und wird zu einem bearbeiteten, umgeformten. Der Arbeitende erfährt im Prozess seine Verfügungsmacht über die Dinge, und er überwindet damit seine unmittelbare Abhängigkeit von ihnen. In seiner Handhabung der Dingwelt vollzieht sich zugleich eine Distanzierung von ihr. Die Arbeit unterbricht die gerade, direkte Gerichtetheit des Triebs auf seine Befriedigung, sie hält an und hält auf. Das spezifische Merkmal der Arbeit ist der Werkzeuggebrauch; mit dem Werkzeug wird etwas zwischen den Trieb und die Natur geschoben.

Hegel bezeichnet das Werkzeug als eine „,vernünftige Mitte “: Es bildet die Mitte zwischen dem passiven Objekt und dem tätigen Subjekt, wobei es an sich selbst

5 Einen Überblick über die Diskussion der Thesen von Habermas bietet Herbert Schnädelbach, Hegels praktische Philosophie, Frankfurt a. M., Suhrkamp Verlag, 2000, S. $155 \mathrm{ff}$.

6 Vgl. ibid., S. 131.

7 Georg Wilhelm Friedrich Hegel, Jenaer Systementwürfe I, Das System der spekulativen Philosophie, neu hrsg. von Klaus Düsıng und Heinz Kimmerle, Hamburg, Felix Meiner Verlag, 1986, S. 209 (im Folgenden zitiert als J I).

8 Georg Wilhelm Friedrich Hegel, Werke in 20 Bänden, Bd. 3: Phänomenologie des Geistes, Suhrkamp Verlag, Frankfurt a. M., 1970, S. 153. 
gleichermaßen Aktivität wie Passivität zeigt. Passiv dienend liegt es dem Subjekt in der Hand, tätig verhält es sich gegenüber dem „toten Ding“. Es ist ferner ein Medium, das den konkreten Arbeitenden und das jeweils Bearbeitete, die einzelnen Arbeitssituationen überdauert und dabei seine eigene Genealogie entwickelt. So bleibt schließlich im Werkzeug die Möglichkeit der arbeitenden Triebbefriedigung als eine allgemeine aufbewahrt.

Das Werkzeug ist die existierende, vernünftige Mitte, existierende Allgemeinheit des praktischen Prozesses, es erscheint auf der Seite des Tätigen gegen das Passive, ist selbst passiv nach der Seite des Arbeitenden und tätig gegen das Bearbeitete. Es ist das, worin das Arbeiten sein Bleiben hat, was von dem Arbeitenden und dem Bearbeiteten allein übrig bleibt und worin die Zufälligkeit sich verewigt; es pflanzt sich in Traditionen fort, indem sowohl das Begehrende als das Begehrte nur als Individuen bestehen und untergehen. ${ }^{9}$

Ist also das Werkzeug an sich bereits ein komplexes Ding, so ist auch sein Gebrauch nicht minder vielschichtig. Der Arbeit wohnt eine besondere Dialektik inne.

Zunächst muss das arbeitende Subjekt sich dem Zwang und dem Eigensinn der Natur unterwerfen. So ist z. B. der zu bearbeitende Boden hart, der Fluss reißend, das Klima widrig. Das Subjekt macht sich in der Unterordnung unter die natürlichen Gegebenheiten selbst zum Ding: es ,ist eben dies“ ein „sich zum Gegenstande machen" ${ }^{\prime 10}$. Aber indem es sich der Eigengesetzlichkeit der Natur unterwirft, wächst dem Ich vermittels des Werkzeuggebrauchs die besondere Erfahrung zu, die Gesetze der Natur kennenzulernen. Durch dieses Wissen kehrt es in einer veränderten Weise, nämlich als ein wissend gewordenes, listiges Bewusstsein aus seiner ersten Verdinglichung in sich selbst zurück.

Das Subjekt hat mittels des Werkzeugs seine Erfahrungen mit der Dingwelt gemacht. Es kann ferner das Werkzeug für sich arbeiten lassen. Das Instrument fungiert als eine Substitution des körperlichen Kraftaufwands, der reduziert und des physischen Verschleißes, der gleichfalls verringert werden kann: „Ich habe die List zwischen mich und die äußere Dingheit hineingestellt, - mich zu schonen und meine Bestimmtheit damit zu bedecken und es [das Werkzeug, F.K.] sich abnutzen zu lassen“. ${ }^{11}$ Das Werkzeug nutzt sich an meiner Stelle ab, dennoch bekomme ich noch „Schwülen“12, d.h. die eigene Verdinglichung im Arbeitsprozess ist nicht vollends aufgehoben.

Die List, die in der Arbeit beschlossen liegt, ist erst mit einem weiteren Schritt vollendet: dann nämlich, wenn das Werkzeug zur Maschine geworden und selbsttätig ist, wie z. B. die Uhrfeder, die Hegel an dieser Stelle nennt, ebenso wie das Wasserrad oder das Windrad. ${ }^{13}$ Wenn Hegel am Rand bemerkt: „die breite Seite der Gewalt wird von der Spitze der List angegriffen"14, so drängt sich unweigerlich das Bild auf, wie mittels der Flügel des Windrads dem an sich blinden Wind die Energieerzeugung abgelistet wird. Wenn die eigene Tätigkeit der Natur ,angewendet wird, um in ihrem

9 J I, S. 211.

${ }^{10}$ J III, S. 189.

11 Ibid.

12 J III, S. 190.

13 Vgl. hierzu auch Hans-Christoph SCHmidt Am Busch, Hegels Begriff der Arbeit, Berlin, Akademie Verlag, 2001, S. 47-58.

14 J III, S. 190. 
sinnlichen Dasein etwas ganz anders zu tun, als sie tun wollte - ihr blindes Tun zu einem zweckmäßigen gemacht wird; - zum Gegenteile ihrer selbst -“"15, dann regiert die List zur Gänze. Der Wille, der zunächst geradeaus gerichteter Trieb war, tritt nun als List „ganz aus der Arbeit zurück; er lässt die Natur sich abreiben, sieht ruhig zu, und regiert nur mit leichter Mühe das Ganze“"16.

Zwei Momente also sind es, die die List kennzeichnen: das Moment der Verkehrung des Richtungssinnes und das Moment der passiven Steuerung. Das praktische Bewusstsein ist also listig darin, dass es in der Arbeit sein Wissen, das es von der Natur erlangt hat, gegen die Natur selbst richtet, um sie für sich arbeiten zu lassen und dabei wesentlich zusehende, steuernde Tätigkeit ist. Die List ist „,von Grund aus Meister, dass das Andere sich in seinem Tun selbst verkehrt" ${ }^{\text {“ }}{ }^{17}$

Der Wille hat sich schließlich im Prozess der Arbeit, im Werkzeug- und Maschinengebrauch entzweit oder anders gesagt, verdoppelt. Der gerade gerichtete, „hinausgehende Trieb“ - von Hegel nun indirekt als männlicher Charakter apostrophiert - ,ist als List theoretisches Zusehen“" und damit weiblicher Charakter geworden, kurz: „Durch die List ist der Willen zum Weiblichen geworden.“"

Die blinde Macht der Natur und der Dinge als Bestimmtheit zu fassen, direkt gegen sie tätig sein, dieser Aspekt des Tätigseins fällt auf die Seite des Mannes - sie aber , an einer Seite anfassen', so, dass die Natur sich gegen sich selbst richtet, und sie als Bewegung eben in sich zurückgehen zu lassen, sie aufzuheben, das ist Sache des Weibes.

Auch die Dynamik der Begierde zwischen den Geschlechtern selbst wird von dieser Form des Gegensatzes bestimmt. So hat „der Mann [...] Begierde, Trieb“, wohingegen dem weiblichen Trieb zukommt, ,vielmehr nur Gegenstand des Triebes zu sein; zu reizen, Trieb zu erwecken, und ihn sich an ihm befriedigen zu lassen. ${ }^{\text {"19 }}$

Dem Übergang von der List im Maschinengebrauch zum Geschlechtsverhältnis liegt demnach folgende Überlegung zugrunde: Geradeso wie ich die Tätigkeit der Maschine nicht mehr als meine erfahre, obwohl ich sie steuere, ohne mich dabei wie in der Ausübung der einfachen Arbeit verdinglichen zu müssen, so ,steuert' in analoger Weise die Frau das sexuelle Verhältnis, indem sie nicht direkte Begierde entfaltet, sondern Begierde nach der Begierde des Mannes hat. Hegel versteht im Weiteren die Geschlechtsbeziehung als die Beziehung dieser polar bestimmten

15 Ibid.

16 Ibid.

${ }^{17}$ Ibid., S. 191.

${ }^{18}$ Ibid., S. 190.

${ }^{19}$ Ibid., S. 191. Dass für Hegel die sittlichen Geschlechtscharaktere bereits in der organischen Natur präformiert sind, zeigt folgende Stelle: „Wie im Manne der Uterus zur bloßen Drüse herabsinkt, so dagegen der männliche Testikel im Eierstocke eingeschlossen, tritt nicht heraus in den Gegensatz, wird nicht für sich, zum tätigen Gehirne, und der Kitzler ist im Weibe das untätige Gefühl überhaupt; das hingegen im Manne das tätige Gefühl, das aufschwellende Herz, [...] - Der Mann ist also das Tätige - dadurch daß diesen Unterschied seine Tätigkeit hat; das Weib aber ist das Empfangende, weil sie in ihrer unentwickelten Einheit bleibt." (J I, S. 161) Hegel folgt auch in der Zeugungstheorie dem traditionellem aristotelisch-galenschen Modell von passiver Materie und aktivem Geist: „Die Zeugung muß man nicht auf den Eierstock und den männlichen Samen reduzieren, als sei das neue Gebilde nur eine Zusammensetzung aus den Formen oder Teilen beider Seiten, sondern im Weiblichen ist wohl das materielle Element, im Manne aber die Subjektivität enthalten." Hegel, Werke in 20 Bänden, Bd. 9: Enzyklopädie der philosophischen Wissenschaften, S. 519. 
Charaktere: der Mann hat einfache Objektbegierde - die Frau hat Metabegierde Begierde nach der Begierde. ${ }^{20}$

\section{Exkurs zu Rousseau}

Auch wenn es etwas gewagt erscheint, zwischen zwei völlig unterschiedlichen philosophischen Ansätzen einfachhin Parallelen zu ziehen, so drängt sich ein Vergleich aus geschlechtertheoretischer Perspektive indes so nachdrücklich auf, dass an dieser Stelle ein kurzer Blick auf Rousseaus Geschlechterkonzeption gestattet sei.

Bei Rousseau weist nämlich die weibliche Scham eine mit der Hegelschen List fast identische Struktur auf. Die Scham ist der zentrale Angelpunkt für die sexuelle Dynamik und die Organisation des Geschlechterverhältnisses. Dabei sind Hemmung, Verkehrung ins Gegenteil, Steuerung und Kontrolle abermals die wesentliche Bestimmung des Weiblichen, näherhin des Weiblichen in sexualibus. Rousseau ist weniger spekulativer Philosoph als Anthropologe, es geht ihm nicht um eine Deduktion der Geschlechtscharaktere aus dem gedoppelten Charakter des praktischen Geistes als vielmehr um eine Konzeption der Geschlechtsidentitäten, die in der Dynamik des Sexualtriebes verankert ist. Aber auch bei Rousseau entstehen die Geschlechtscharaktere aus einem komplexen Ineinander von Trieb und Hemmung, auch hier geht es um eine Kultivierung der Stillung der Begierde, die menschlich wird durch die Hemmung. Trotzdem ist der Unterscheid klar: Hegel gewinnt die geschlechtliche Differenzierung im Geistigen aus dem Erfahrungsprozess, den das Bewusstseins in der Arbeit durchläuft, Rousseau hingegen zeigt, wie die „moralischen“ Geschlechtsidentitäten sich aus ihren ,physischen“ Voraussetzungen herausentwickeln, er verfolgt ,wie uns das Physische unmerklich zum Moralischen führt ${ }^{\text {‘21 }}$. Die Überlegungen sind dabei folgende: Animalisches Paarungsverhalten folgt instinktverankerten Mustern; diese steuern die periodische Aktualisierung des Fortpflanzungstriebes und begrenzen ihn auf ein am Erhalt der Gattung orientiertes Maß. Die menschliche Sexualität hingegen muss wegen der ihr inhärenten Maßlosigkeit anderen Formen von Regulierung unterworfen werden. Der Scham fällt die Rolle zu, das auf Permanenz gestellte Erregungspotential des Menschen in eins zu aktualisieren und zu begrenzen, d.h. zu steuern und zu kontrollieren. Dabei bildet die weibliche Scham das initiierende Moment in der sexuellen Dynamik, insofern sie das männliche Verlangen ,entflammt [...], indem sie es hemmt" ${ }^{\text {“22 }}$. Die Frau hat allerdings mittels der Scham zunächst ihre eigenen spontanen Triebregungen gehemmt und in der Folge mit ihrem widerstrebenden Verhalten das männliche Begehren allererst evoziert. Hier findet man also die gleichen Charaktere, wie sie die als weiblich konnotierte List bei Hegel aufweist: das rekursive Moment des InSich-Zurückgehens und das der Steuerung.

\footnotetext{
20 Vgl. Andreas WiLDT, Autonomie und Anerkennung, Stuttgart, Verlag Klett-Cotta, 1998, S. 354f.

${ }^{21}$ Jean-Jacques Rousseau, Emil oder Über die Erziehung, Paderborn/ München/ Wien/ Zürich, Schöningh, 1993, S. 389.

22 Jean-Jacques Rousseau, Brief an d'Alembert über das Schauspiel, in ders., Schriften, Bd. 1, Frankfurt a. M., Fischer Taschenbuch Verlag, S. 419.
} 
Nun ist aber der Charakter des Weiblichen an sich nicht spekulativ aus dem praktischen Geistesvermögen deduziert worden, sondern Männer und Frauen sind bei Rousseau schlicht vorausgesetzt. Vor dem Hintergrund bleibt bei Rousseau die Frage zu beantworten, warum die Figur der aktiven Passivität oder der passiven Aktivität an die Frau gebunden ist, warum die Scham als die Form der sexuellen List überhaupt auf die Seite der Frau fällt? Der Grund liegt im Physischen, nämlich in der asymmetrischen Verteilung der sexuellen Potenz zwischen Mann und Frau. Das männliche Geschlechtsvermögen ist fragil, insofern als Wollen und Können, Begehren und Potenz, nicht immer und nicht notwendig übereinstimmen. Dagegen ist die weibliche Begierde unbegrenzt. Weil die Frau den sexuellen Akt immer vollziehen kann, werden ihr - ein Topos des 18. Jahrhunderts - „unbegrenzte Begierden" ${ }^{23}$ unterstellt. Räumte man nun beiden Geschlechtern gleichermaßen die Möglichkeit zur unverstellten sexuellen Initiative ein, würden aufgrund der ungleichgewichtigen Voraussetzungen „Können und Wollen nie übereinstimmen und die Begierden nie einander entsprechen“"24. Die Scham als die natürliche Tugend der Frau reguliert somit die „unbegrenzte[n] Begierden“"25 der Frau und provoziert mit ihrem Widerstand allererst das Begehren des Mannes.

Die Verantwortung für eine gleichermaßen natürliche wie kultivierte Gestaltung der menschlichen Geschlechtlichkeit liegt demnach bei der Frau: Ihr obliegt die Initiative in einer passiven Form, d.h. sie ist dazu bestimmt, dem Mann zu gefallen, ihn zu erregen, und sie hat die Aufgabe, die geschlechtlichen Triebpotentiale beider Geschlechter zu begrenzen und zu steuern. Ein Unterschied in der Konzeption des Triebgeschehens lässt sich freilich bei den beiden Autoren feststellen: während bei Rousseau die Frau auch noch ihre eigene „unbegrenzte“ Begierde steuern muss, so hat sie bei Hegel nur noch Metabegierde, also den Trieb in der Form, dass er darauf zielt „nur Gegenstand des Triebes“ zu sein.

Nach diesem Ausblick zurück zu Hegel. Erinnern wir uns: Die Entzweiung des Willens in zwei Charaktere ist Grundlage der Geschlechtsdifferenz, die sich folgendermaßen ausbuchstabieren lässt: das Männliche behauptet sich als die Macht der Entgegensetzung, als das geradeaus Gehende, Hinaustreibende und Getriebene, das andere regiert als das Weibliche: als das in sich Seiende, Unterirdische, das so gegen das Seiende tätig ist, dass es dessen Selbsterhaltung das Negative unterschiebt. Ein Resümee des Übergangs vom Werkzeuggebrauch liest sich schließlich im Jenaer Systementwurf I von 1803/04 wie folgt:

[...] indem es [das Bewusstsein F.K] in der Arbeit zur Mitte des Werkzeugs wird, indem es [...] hier seine reale Herrschaft gegen die Natur sich erwiesen und damit sich als für sich der Natur entnommener Geist konstituiert und sich für sich gestaltet, den Gegensatz nach außen aufgehoben hat; so zerfällt es in sich selbst und realisiert [sich] in gegeneinander differierenden Momenten, deren jeder selbst ein Bewusstsein ist, in der Geschlechtsdifferenz, in welcher es ebenso die einzelne Begierde der Natur aufhebt und zur bleibenden Neigung macht. ${ }^{26}$

\footnotetext{
23 RoussEau, Emil oder Über die Erziehung, S. 387.

${ }^{24}$ Rousseau, Brief an d'Alembert über das Schauspiel, S. 419.

25 RoussEau, Emil oder Über die Erziehung, S. 387.

${ }^{26}$ J I, S. 196.
} 
Systematisch stiftet die Entzweiung des Willens in die geschlechtlichen Charaktere den Übergang von der Arbeit zur Familie, in der Diktion von Habermas: vom instrumentellen Handeln zur Interaktion. Die These Hegels ist, dass, insofern in der Geschlechtsdifferenz die natürliche Begierde zur bleibenden Neigung aufgehoben ist, der Trieb sich auf der Grundlage des Charakters in Liebe verwandelt hat. ${ }^{27}$

An die Stelle des Verhältnisses von Begierde und ,, totem Ding “ tritt nun ein Verhältnis eines individuellen Bewusstseins zu einem anderen, wobei jedes Bewusstsein sich im anderen selbst anschaut. Hegel umreißt in diesen Abschnitten zum Geschlechterverhältnis und zur Familie Vorformen des Konzepts der Anerkennung als dem Grundprinzip von Sittlichkeit überhaupt. Im Bereich der natürlichen Sittlichkeit der Familie findet jedoch noch keine Anerkennung, sondern nur ein Erkennen statt. Anerkennung ist demgegenüber eine Form von intersubjektivem Verhältnis, wie sie nur außerhalb familialer Verhältnisse besteht. ${ }^{28}$ Dieser geschlechtertheoretisch bedeutsame Unterschied von Erkennen und Anerkennen kann hier nur angedeutet werden.

Erkennen als Grundbegriff des Geschlechterverhältnisses nimmt nach Hegel die sexuelle Konnotation biblischer Herkunft mit auf, sodass mit Erkennen neben der Selbstwahrnehmung im Anderen auch der Vollzug des Geschlechtsverkehrs mitbezeichnet ist.

Im geschlechtlichen Erkennen wird der natürliche Trieb versittlicht: „Dies Erkennen ist die Liebe. “29 Gleichwohl ist das Erkennen von Mann und Frau nur „Element der Sittlichkeit, noch nicht sie selbst, es ist nur die Ahndung derselben.“30 Das wechselseitige Erkennen der Geschlechter ist gerade noch nicht die wechselseitige Anerkennung zweier ausdifferenzierter Selbstheiten, sondern das Erkennen von zwei Extremen, die sich entgegengesetzt sind, insofern das eine innerlich das ist, was das andere äußerlich ist: „[...] jedes ist darin dem Anderen gleich, worin es sich ihm entgegengesetzt hat. ${ }^{\text {" }}{ }^{31}$ Im Gegensatz dazu bedeutet Anerkennen indes ein Erkennen des anderen Bewusstseins nicht mehr nur als „Charakter“, von dem man sich unterscheidet und mit dem man sich doch zugleich in der Liebe eins weiß, sondern als eine Verkörperung derselben Totalität, die man selbst ist. ${ }^{32}$

\section{Das Böse}

Kehren wir nach diesem Ausblick auf den systematischen Fortgang des Textes noch einmal ins Zentrum der Analyse des Werkzeuggebrauchs zurück. Durch die Dialektik der Arbeit und im Medium von Werkzeug und Maschine ist der

27 Vgl. SchnäDELBACH, Hegels praktische Philosophie, S. 131.

28 Vgl. ibid., S. 151.

29 J III, S. 192.

30 Ibid.

31 Ibid.: „Dies Erkennen ist nur Erkennen der Charaktere; oder beide haben sich gegeneinander noch nicht als Selbst bestimmt, nur das Eine ist das Wissen, in sich, und das Andere Wissen als Tätigkeit nach außen".

32 Vgl. J III, S. $193 f$. 
Willen, wie gezeigt, ,ein gedoppelter entzweiter ${ }^{“ 33}$ geworden. Er hat sich in ,zwei Extreme entzweit" ${ }^{\star 34}$ und dabei zwei Charaktere ausgebildet. Der eine Charakter ist zielgerichtet, intentional auf die Sache gerichtet, geradeaus gehend', aber gewissermaßen auch blind. Der andere Charakter ist in sich verbleibend, wissend und zusehend, untergründig.

Diesen inneren, distanziert-wissenden, weiblichen Charakter, der gegen das Seiende so tätig ist, dass es ,seiner Selbsterhaltung das Negative unterschiebt', bezeichnet Hegel nun auch als das Böse. Der Begriff des Bösen taucht unvermittelt auf und wird in Zusammenhang des Vorlesungsfragments nicht weiter erläutert. Allerdings widmet Hegel dem Phänomen des „Bösen“ in den Vorlesungen über die Philosophie der Religion längere Passagen. Aus diesen erschließt sich, dass „böse“ in einem fundamentalen und gewissermaßen moralisch neutralen Sinn zu verstehen ist.

Der Mensch muss „böse“ werden, er muss die Natur verlassen, um das zu werden, was er seiner Natur nach ist: freier Geist. Der Mensch ist notwendigerweise böse, da er als freier Geist die Möglichkeit hat gut oder böse zu handeln. Dieses Böse-Sein ist gleichsam die Bedingung der Möglichkeit der Erkenntnis von Gut und Böse und zu gutem und bösem Handeln. Damit hat man, wie sich unschwer erkennen lässt, im Kern eine spekulative Deutung des Sündenfalls.

Nach Hegel markiert der Sündenfall die Entzweiung des Geistes gegenüber der Natur.

Der Geist ist nur wozu er sich macht; er muss, um sich selbst zu realisieren, in die Entgegensetzung gehen. Der Mensch ist Geist im Wissen und Wollen dessen was das Rechte ist und eben dieses Wissen und Wollen setzt die Differenz des Bewusstseins von sich selbst voraus. ${ }^{35}$ Das Böse hat wie das Gute im Willen seinen Ursprung. ${ }^{36}$ Der natürliche Wille ist an sich der Widerspruch, sich von sich selbst zu unterscheiden. Das in sich Entzweite, das Gedoppelte des Willens ist allererst die Voraussetzung für Gut und Böse. Die Freiheit zur Verantwortlichkeit liegt in der Umkehrung des Geistes in sich selbst, die dem Geist wesentlich ist, begründet.

Wie lassen sich die religionsphilosophischen Überlegungen nun näher an den oben entfalteten Gedanken des listigen Bewusstseins anschließen? Hegel zufolge wird in der Genesis in Form einer Geschichte erzählt, also in ein zeitliches Nacheinander gebracht, was sich philosophisch als systematischer Zusammenhang darstellt. ${ }^{37}$ Die

33 J III, S. 191.

34 Ibid.

35 „So weiß er erst von Gott und dem Guten; wenn er davon weiß, hat er es zum Gegenstand seines Bewußtseins; hat er es zum Gegenstand seines Bewußtseins, so unterscheidet sich das Individuum davon." Hegel, Werke in 20 Bänden, Bd. 16: Vorlesungen über die Philosophie der Religion I, S. 265.

36 „Der natürliche Wille ist an sich der Widerspruch, sich von sich selbst zu unterscheiden, für sich und innerlich zu sein. Wenn man sagt, das Böse enthält die nähere Bestimmung, dass der Mensch böse ist, insofern er natürlicher Wille ist, so würde dies der gewöhnlichen Vorstellung entgegengesetzt sein, welche sich gerade den natürlichen Willen als den unschuldigen und guten denkt." Hegel, Werke in 20 Bänden, Bd. 7: Grundlinien der Philosophie des Rechts, S. 264.

37 „Hierüber ist uns eine alte Vorstellung, der Sündenfall, in der Bibel aufbewahrt. Diese bekannte Darstellung, wie das Böse in die Welt gekommen, ist in die Form eines Mythos, einer Parabel gleichsam eingekleidet. Wenn nun das Spekulative, das Wahrhafte so in sinnlicher Gestaltung, in der Weise von Geschehensein dargestellt wird, so kann es nicht fehlen, dass unpassende Züge darin vorkommen.“ Hegel, Werke in 20 Bänden, Bd. 17: Vorlesungen über die Philosophie der Religion II, S. 75. 
biblische Erzählung nimmt ihren Ausgang im Paradies, welches die Unentzweitheit von Mensch und Natur abbildet, in der Folge ereignet sich der Sündenfall als eine Art „Unfall“. Nach dem Sturz aus der paradiesischen Unschuld haben Adam und Eva ein Wissen um Gut und Böse, sie erkennen sich allererst als Mann und als Frau, und es ist ihr Menschenschicksal geworden, dass sie ihr Dasein durch ihrer Hände Arbeit fristen müssen.

Für die spekulative Philosophie ist freilich der Verlust der ersten unschuldigen Ungeschiedenheit kein ,äußerlicher“ Unfall, sondern die Entzweiung macht gerade die Notwendigkeit des Geistes aus: „Dass das Paradies verloren ist, zeigt uns, dass es nicht absolut als Zustand wesentlich ist. “38 Dementsprechend ist auch die Arbeit nicht die Folge, sondern eben die Realisation des Austritts aus der natürlichen Ungeschiedenheit. In der Arbeit hebt der Mensch das, ,was ihm natürlicherweise notwendig ist, zu einer Sache seiner Freiheit empor." ${ }^{\text {"39 }}$ Im Medium der Arbeit und des Werkzeuggebrauchs erzeugt der Wille sich in einer zwiefältigen Gestalt, er bildet einen männlichen und weiblichen Charakter aus: die Geschlechtsdifferenz tritt hervor. Und bei all dem ist schließlich der Grund der Freiheit, das „Böse“, das innerlich zu sich zurückkehrende, das „umkehrende“ Moment des Willens auf die Seite des Weiblichen gefallen. Es zeigt sich mithin, dass in die kleine Passage zur List und dem Weiblichen aus dem Systemfragment von 1805/06 in gewisser Weise das große Bild vom Sündenfall eingeschrieben ist.

\section{Die ewige Ironie des Gemeinwesens}

An dem systematischen Zusammenhang von Trieb und Befriedigung, Arbeit und Werkzeug mit der Differenzierung des Willens in einen männlichen und weiblichen Charakter und dem sich anschließenden Übergang zu Geschlechtsliebe, Ehe und Familie hat Hegel über die frühen Vorlesungsfragmente hinaus nicht festgehalten.

Allerdings findet man Teile dieser vorangegangenen Reflexionen in der Phänomenologie des Geistes in einen veränderten systematischen Aufriss eingeordnet. So wird die emanzipatorische Potenz der Arbeit im wohl berühmtesten Abschnitt des Werks, im Herr-Knecht-Kapitel entfaltet, ohne dass dort allerdings die List und die Geschlechtscharaktere ins Spiel kommen. Der Themenkomplex Geschlechtscharakter, Verhältnis der Geschlechter und Familie wird hingegen an einer späteren Stelle der Phänomenologie ausführlich dargestellt, und zwar dort, wo Hegel die erste Form der Sittlichkeit, den Geist als die unmittelbare Einheit von Selbstbewusstsein und Gemeinbewusstsein, von Individuum und Gemeinschaft behandelt und in der realen historischen Gestalt der griechischen Polissittlichkeit aufsucht.

Das „schöne sittliche Leben“ der Griechen besteht im Verhältnis zweier Arten von Gesetz und ihrer Herrschaftsbereiche. Die Sittlichkeit teilt sich in zwei Formen: auf der einen Seite das positive menschliche Gesetz und auf der anderen

\footnotetext{
${ }^{38}$ Hegel, Werke in 20 Bänden, Bd. 16: Vorlesungen über die Philosophie der Religion I, S. 267.

39 Hegel, Werke in 20 Bänden, Bd. 17: Vorlesungen über die Philosophie der Religion II, S. 77.
} 
das ungeschriebene - Hegel sagt auch: unterirdische - Gesetz der Götter. Für die Bewahrung des einen steht der Staat, für die des anderen die Familie. Der griechische Geist ist wesentlich eine Stufe des Übergangs, er ist das „Herüberwenden des Natürlichen zum Geistigen“"40. In der Phänomenologie des Geistes arbeitet Hegel besonders deutlich heraus, dass das Heraustreten des Geistes aus der Natur und die Etablierung von Rechtsverhältnissen in der griechische Antike notwendig mit einem Konflikt zwischen den familiären Strukturen einerseits und dem in seiner Entwicklung begriffenen politischen Willen andererseits einhergehen. ${ }^{41}$

Dieser Konflikt zwischen dem ,menschlichen' und dem ,göttlichen' Gesetz, zwischen Männern und Frauen, wird nach Hegel vorbildlich in den antiken Tragödien dargestellt, insbesondere in Sophokles'Antigone. Kreons Anspruch, familiäre Belange den Interessen der Polis unterzuordnen, ist nach Hegel gerechtfertigt, denn er vertritt damit das Prinzip, auf das der Geist sich hin entwickeln muss: er muss sich selbst bewusst bestimmen und beherrschen lernen um der Freiheit willen. Aber es gelingt Kreon nicht, zugleich die Berechtigung der Familienpietät zu erfassen. Denn es ist die Erinnerung, die Bewahrung des toten Mitglieds, welche die Familie zu einem sittlichen Zusammenhang macht und damit über einen rein natürlichen Zusammenhang der wechselseitigen Bedürfnisbefriedigung hinaushebt.

In diesem Kontext begegnet man der Verknüpfung von List, Verkehrung und weiblichem Charakter wieder. Freilich in einer vorderhand etwas modifizierten Form, denn Hegel spricht mit Blick auf die Rolle der Frauen im antiken Staat von Intrige und in einer grundsätzlichen Weise von einer „ewigen Ironie des Gemeinwesens“. Was ist nun hier gemeint?

Männerwelt und Frauenwelt, Staat und Familie, Polisgesetze und Familienpietät stehen durch ihre jeweilige Einseitigkeit in einem permanenten ungelösten Konflikt miteinander. Der wesentliche Zweck der Familie ist der Einzelne. Insofern nun der Staat das Prinzip des Einzelnen um des Allgemeinen willen unterdrückt und unterdrücken muss, macht im Gegenzug das Niedergehaltene sich geltend in Form der Intrige. Da die Frauen keine öffentlichen, d.h. nicht geradeweg ,offen heraus' Ansprüche erheben können, agieren sie untergründig gegen den Staat. Gegen den allgemeinen Staatszweck befördern die Frauen die Feier einzelner Individuen, nämlich ihrer Söhne. Der Staat wiederum bedarf der großen Einzelnen für seine Selbstverteidigung bzw. für den Krieg. Er ist damit abhängig von den großen Söhnen und fällt schließlich dem Heroenkult anheim. Das Prinzip der Einzelheit siegt letztendlich über das staatliche Prinzip des Allgemeinen: "der tapfere Jüngling, an welchem die Weiblichkeit ihre Lust hat, das unterdrückte Prinzip des Verderbens tritt an den Tag und ist das Geltende. “42

Eben diese abermals „innerlich“, „im Verborgenen“ untergründig lancierte Verkehrung ins Gegenteil, im Falle der antiken Sittlichkeit diejenige von Einzelheit und Allgemeinheit, nennt Hegel die „ewige Ironie des Gemeinwesens“. Es ist der

${ }^{40}$ Hegel, Werke in 20 Bänden, Bd. 12: Vorlesungen über die Philosophie der Geschichte, S. 301.

${ }^{41}$ Vgl. Eva Bockenheimer, Hegels Familien- und Geschlechtertheorie, Felix Meiner Verlag, Hamburg, 2013, S. 48f.

${ }^{42}$ Hegel, Werke in 20 Bänden, Bd. 3: Phänomenologie des Geistes, S. 315. 
immanente, unauflösliche Widerspruch in der antiken Polis, der ihren Untergang besiegelt. ,Ironisch' ist es, dass das Gemeinwesen, gerade weil es das Prinzip der Einzelheit der Familie unterdrückt und unterdrücken muss, dieses Prinzip selbst mit erzeugt und durch diese Unterdrückung sogar zusätzlich anstachelt. ${ }^{43}$ In den Jenenser Systementwürfen hatte Hegel diese Bewegung „dass das Andere sich in seinem Tun selbst verkehrt" als List und als das Weibliche des Willens charakterisiert. Auf dem Feld des objektiven Geistes in der Form der griechischen Sittlichkeit erscheint diese Figur einer inneren ,intriganten“ Umkehrung und Verkehrung ins Gegenteil abermals und diesmal realiter an die Frauen als Protagonistinnen geknüpft. Ironischerweise bringt das Gemeinwesen immerwährend - „ewig“ - auch das mit hervor, was sich gerade gegen das Prinzip des Staates richtet und es unterminiert, hier die Apotheose des Einzelnen. Diese innere Gegenläufigkeit besiegelt schließlich den Untergang der griechischen Sittlichkeit.

${ }^{43}$ Vgl. BocKenheImer, Hegels Familien- und Geschlechtertheorie, S. 424. 